\title{
IV.
}

(Aus der Nervenklinik des Krankenhauses zum hl. Geist und aus dem Laboratorium von Dr. E. Flatau in Warschau.)

\section{Ein Fall von Syphilis des Rückenmarks und seiner Hänte in der Lumbosacralgegend (Meningomyelitis lumbosacralis syphilitica) mit ungewöhnlichen trophischen Störungen. \\ Von}

\author{
Dr. St. Kopezyński, \\ ehem. ordin. Arzt der Nervenklinik der Universität in Warschan.
}

(Nach einem im Warschaner Aerzteverein 4. II. 02 gehaltenen Vortrage.)

(Mit 6 Abbildungen im Text und Tafel II.)

Die Syphilis des Nervensystems gehört zu den Erkrankungen, welchen bedeutsame Kliniker und Neurologen viel Zeit und Aufmerksamkeit geopfert haben, angeregt einerseits durch den ausserordentlichen Polymorphismus des Leidens, andererseits dadurch, dass es nahezu das einzige Gebiet der Neuropathologie ist, wo eine frühzeitige Diagnose und eine richtige Behandlung von grösstem Nutzen sein können.

Seit der klassischen Arbeit Heubner's') sind zahlreiche mehr oder weniger werthvolle Abhandlungen publicirt worden über die Syphilis des Nervensystems, darunter in den letzten 15 Jahren mehrere bedeutende Monographien von Oppenheim ${ }^{2}$ ), Rumpf ${ }^{3}$ ) und zuletzt die umfangreiche Arbeit von Nonne $e^{4}$ ), dessen Literaturverzeichniss über 1000 Arbeiten enthält.

In der polnischen Literatur haben nur wenige Autoren über die Syphilis des Nervensystems geschrieben, aber diese Arbeiten haben

1) Heubner, Die luetische Erkrankung der Hirnarterien. Leipzig 1874.

2) Oppenheim, Die syphilitischen Erkrankungen des Gehirns. (Bei Nothnagel.) Wien 1897.

3) Rumpf, Die syphilitischen Erkrankungen des Nervenssystems. Wiesbaden 1887.

4) Nonne, Syphilis und Nervensystem. Berlin 1902.

Deutsche Zeitschr. f. Nervenheilkunde. XXIV. Bd. 
für diesen Gegenstand einen bedeutsamen Beitrag geliefert. Abgesehen vom Sammelreferat von Dr. Fabian, haben wir hier die Arbeiten von Domański1), Gajkiewicz, Goldflam und Orlowski za nennen.

Gajkiewicz'2) beweist an der Hand von 50 Fällen den Polymorphismus der Syphilis des Nervensystems und giebt klinische Bilder dieser Krankheit, namentlich für den praktischen Arzt vom grössten diagnostischen Werth.

Goldflam ${ }^{3}$ ) behandelt die Syphilis des Rückenmarks in einer klinischen Vorlesung mit hervorràgender Vielseitigkeit und Kriticismus. Er giebt 13 eigene Beobachtungen mit verschiedener Localisation des Leidens, darunter 3 mit ausführlicher mikroskopischer Untersuchung.

St. Orlowski4) behandelt in einer erschöpfenden Monographie die Entwicklung und den jetzigen Stand der Lehre von der Rückenmarkssyphilis an der Hand eines umfangreichen Materials (60 Fälle, darunter 26 eigene Beobachtungen und 5 mikroskopische Untersuchungen).

Die Wichtigkeit des Gegenstandes soll mich entschuldigen, wenn ich zu diesen Monographien die Beschreibung eines Falles hinzufüge, welcher mir in klinischer und pathologisch-anatomiseher Hinsicht von Interesse zu sein scheint. Es handelt sich hier um eine seltene Localisation der Syphilis im untersten Abschnitt des Rückenmarks mit intravital beobachteten ungewöhnlichen trophischen Störungen.

Emilie J., 20 Jahre alt, Näherin, unverheirathet, kam in die Nervenklinik des Krankenhanses zum heiligen Geist am 26. I. 01 and klagte über Schmerzen and Einschlafen der Beine, Schmerzen in der Brust, Schwäche der Beine, so dass sie nicht gehen konnte, Kopfschmerzen, Herzklopfen, Zusammenschnüren im Halse. Sie fühlt sich krank seit mehreren Monaten. Im November und December war sie schon in demselben Krankenhause auf der inneren Abtheilung wegen allgemeiner Körperschwäche. Um sich zu stärken trank sie in dieser Zeit mehrere Flaschen Wein und Cognac. Der Zustand wurde aber trotzdem immer schlimmer. Vor der

1) Domaŕski, O kile ukladu nerwowego. (Syphilis des Nervensystems.) Przegl. Lek. Krakau 1880.

2) Gajkiewicz, Syfilis ukladu nerwowego. (Syphilis des Nervensystems.) Warsch. klin. Vorträge. Nr. 18-20. 1891.

3) Goldflam, O przymiocie rdzenia (Rückenmarkssyphilis.) Warsch. klin. Vorträge. Nr. 32-33. 1891. 1898.

4) St. Orlowski, Syphilis rdzenia (Rückenmarkssyphilis.) Warschau 
Krankheit hielt sie sich immer für eine sehr nervöse Person: sie weinte oder lachte sehr leicht ohne besonderen Anlass u. s. w. Im Allgemeinen war sie aber immer gesund; sie hat keine Infection durchgemacht. Die Todesursache des Vaters war ihr unbekannt; die Mutter lebt und ist gesund. Geschwister hat sie nicht.

Objective Untersuchang: Mittelgross, mässig gebaut und genährt. Haut und Schleimhäute blässlich. Puls 80, Körperwärme normal. An den Brust- und Bauchorganen nichts Abnormes. Schädel normal, beim Beklopfen nicht schmerzhaft. Wirbelsäule auch normal. Der Gang ist äusserst erschwert, auch wenn die Kranke unter den Armen gestützt wird, angeblich wegen Schmerzen und Schwäche der Beine. Ataxie ist beim Gehen nicht zu finden. Die rohe Muskelkraft ist in allen Extremitäten abgeschwächt, besonders in den unteren, aber ohne deutliche Lähmung einzelner Muskelgruppen. Ataxie der Arme ist nicht vorhanden. Leichte Abnahme des Muskeltonus in sämmtlichen Extremitäten.

Contracturen oder fibrilläre Zuckungen nirgends zu sehen. Sehnenreflexe in den oberen Extremitäten abgeschwächt, Patellar- und Achillessehnenreflexe fehlen vollständig. Hautreflexe schwach. Auf dem ganzen Körper zerstreute „Plaques analgésiques, anesthétiques" ohne besondere Localisation; mitunter Abnahme der Sensibilität auf der ganzen rechten Körperhälfte. Bedeutende Druckempfindlichkeit der Nervenstämme, besonders der Nn. ischiadici. Diese Schmerzhaftigkeit soll der Kranken die Bewegungen erschweren. Die elektrische Untersuchung der Nerven und Muskeln ergab keine Entartungsreaction. Diese Untersuchung wurde aber erheblich erschwert wegen grosser Schmerzen auch bei schwachen Strömen. Die Kopfnerven wiesen nichts Abnormes auf. Pupillen gleich weit, reagiren prompt auf Lichteinfall und Accomodation. Sie sieht und hört gut. Stuhl- und Harnabgang normal. Der Harn ist eiweiss- und zuckerfrei.

Es wurde Brom und Salicyl und warme Salzbäder 3 mal wöchentlich verordnet. In den folgenden Tagen wurden die Schmerzen besonders in den Beinen geringer. Die Muskelkraft, namentlich in den Armen, besserte sich. Die Kranke konnte aber nicht gehen, äusserte noch mancherlei Klagen, bekam mehrmals Weinkrämpfe, scheinbar ohne besondere Ursache, einmal sogar hatte sie einen hysterischen Anfall mit Zuckungen.

4. IV. 01. Die Kranke kann den Hain nicht halten and lässt ihn, ohne es zu merken, unter sich. Da ihre Nachbarin, die an Tabes litt, schon längst den Harn unter sich laufen liess, haben wir anfänglich an die Möglichkeit einer Nachahmung gedacht. Nach geringen Schwankungen stellte sich aber die Incontinenz dauernd ein. Ueber Schmerzen klagt die Kranke nicht.

11. IV. 01. Die Beine werden dick. Im Harn Spuren Eiweiss, keine Cylinder. Abends Temperatursteigerungen. Keine Schmerzen. Bewegungen der Beine besser.

15. IV. 01. Auf den Hinterbacken zeigen sich Decubitusgeschwüre, die trotz Verband nicht heilen wollen wegen Durchnässung mit Harn. Es stellen sich pyämische Fieberschwankungen ein $\left(37^{0}-39,6^{\circ}\right)$. Zunge trocken. Abends Schüttelfrost. Puls 120. Stuhl, retinirt seit 8 Tagen, erfolgte nach Klysma.

22. IV. 01. Auf der Innenseite beider Halluces and auf den Fersen 
umschriebene Röthung (Erythem) und später Blasen mit serösem Inhalt, die nachträglich zu schwarzen brandigen Wunden werden, welche an den Fersen tief bis zum Knochen hineindringen. Die Kranke klagt über fliegenden Schmerz an verschiedenen Körperstellen. Die Decubitusgeschwüre auf den Hinterbacken werden immer breiter und dringen tief bis zum Knochen hinein. Die Wunden werden täglich verbunden.

6. V. 01. Der Zustand wird immer schwerer. Puls 130. Temperatur 36,4-38-40,20. Aehnliche Brandwunden entstehen in derselben Reihenfolge (Erythema, Bulla, Gangraena) an anderen Körperstellen: auf der Aussenseite der Unterschenkel dicht am Malleolus; die Ulceration hat rechts $3-4 \mathrm{~cm}$ Durchmesser, links $2-3 \mathrm{~cm}$; ähnliche kleinere Geschwüre auf der Aussenseite der Füsse, auf der Innenseite der Unterschenkel gleich unter dem Knie 5-6. cm Durchmesser. Die Geschwüre sind fast symmetrisch, dringen $3-4 \mathrm{~cm}$ tief in den Körper, hie und da sind in ihnen brandige schwarze Nervenstämme und Gefässe zu sehen. Nur 2 grosse Geschwüre in der Gegend der Leistenbenge haben keine Symmetrie auf der linken Seite. Wegen grossen Schmerzen bekommt die Patientin Morphium mit Coffein. Das Bewusstsein ist rollkommen erhalten.

18. V. 01. Zustand sehr schwer. Die Geschwüre haben gar keine Neigung zur Heilung. Die Fieberschwankungen sind unverändert. Zunge trocken. Schüttelfrost. Die Pupillen reagiren träge auf Lichteinfall. Die Sehnervpapillen sind leicht hyperämisch (Neuritis optica).

30. V. 01. Puls 140, sehr klein. Temperatur $37-40^{\circ}$. Es hat sich Husten eingestellt. Bewusstsein immer vollkommen klar. Die Kranke klagt über Kälteempfindung. Das Verbinden der Geschwüre ist wegen Schmerzen sehr peinlich, wird aber täglich von Chirurgen gemacht. Auf den Hinterbacken ist der Knochen auf grossen Flächen entblösst. Die Geschwüre verbreiten einen sehr üblen Geruch. Innerlich wird Ipecacuanha mit Coffein verordnet, ausserdem wird Campheröl zweimal täglich eingespritzt.

10. VI. 01. Puls 146, T. 37-39,20. Bewusstsein klar. Extreme Schwäche. Untersuchung unmöglich wegen starker Schmerzen bei der leisesten Berührung.

11. VI. 01. Exitus letalis.

Die Autopsie wurde nach 24 Stunden ansgeführt. Die Leiche ist etwas abgemagert, Haut und Schleimhäute blass. Die Hant zeigt an den oben genannten Stellen, d. h. an den Hinterbacken, Füssen, Unter- und Oberschenkeln die im Decursus morbi geschilderten riesigen Geschwüre, wie sie dem Prosector noch niemals von solcher Grösse zu Gesicht gekommen sind (s. Tafel II). Im rechten unteren Lungenlappen kachektische Pneumonie.

Das Herz klein. Aorta eng. Myo- und Endocardium anämisch. Die Milz gross, hart (Tumor lienis chronicus). Offuscatio parenchymatosa hepatis et renum.

Im Colon katarrhalische Entzündang mit punktförmigen Ekchymosen. Der Schädel quadratisch. Die Hirnhäute sind leicht hyperämisch. Die Gefässe der Hirnbasis ohne makroskopische Veränderungen. Die Gehirnsubstanz anämisch, ohne makroskopische Veränderungen. Nach der Oeffnung des Rückenmarkkanals sieht man an den Knochen und auf der Dura eine hochgradige Hyperämie; hie und da, besonders im Brusttheil geronnene 
Blutextravasate. Die Substanz des Rückenmarks zeigt auf den Querschnitten im Brusttheil vollkommen normale Configuration, nur in der Lendenschwellung sind die Conturen der grauen Substanz verschwommen and das Rückenmark selbst hat eine abnorme gelbliche Verfürbung.

Das herausgenommene Rückenmark und herausgeschnittene Stücke der Nn. tibialis dexter et sinister und peroneus sinister wurden in Müller'scher Lösung gehärtet. Einzelne Rückenmark- und Nervenstückchen wurden mit Osmiumsäure behandelt. Zum Einbetten wurde Celloidin verwendet. Gefärbt wurde nach Weigert-Pal, van Gieson, Marchi mit Carmin und Alaunhämatoxylin. Es wurden Serienschnitte von den untersten Rückenmarksabschnitten (Conus medullaris und Cauda equina) bis zum sechsten Dorsalsegment angefertigt.

Die Ergebnisse der mikroskopischen Untersuchung gestallteten sich folgendermassen.

N. tibialis sinister. An Quer- und Längsschnitten, nach Marchi gefärbt, sieht man sehr bedeutende Veränderungen: Grössere compacte Klumpen Myelin sind nicht zu sehen, man findet dagegen zahlreiche zerstreute Körnchen oder vielmehr Klümpchen, die aus kleinen runden Körnchen, bestehen. Das mikroskopische Bild zeigt auch die weiteren Stadien des Processes, wo die Resorption des zerfallenen Myelins erfolgte. Alle diese Veränderungen beziehen sich auf die Nervenbündel (Fig. 1 u. 2 S. 182 u. 183).

Nach Weigert. Die Veränderungen in den Nerven sind nahezu dieselben. Nerven mit erhaltenen Myelinfibrillen sieht man garnicht. Es sind nur kleine zerstreute Myelinklümpchen geblieben von rundlicher oder länglicher Form, welche aus runden Körnchen zusammengesetzt sind; hie und da sieht man dunkle Klümpchen von mehr homogener, scheinbar concentrischer Structur. Das deutet auf ein früheres Degenerationsstadium hin. Neben diesen grösseren Klümpchen sieht man auf dem ganzen Querschnitt in jedem Bündel eine grosse Anzahl kleiner runder Körnchen. Auf dem Längsschnitt sieht man gleichfalls keine normalen Nervenfasern, sondern längliche im Zickzack verlaufende Streifen, welche aus rosenkranzähnlich gruppirten Klümpchen von zerfallenem Myelin zusammengesetzt sind. Doch sieht man hier auch ein Uebergewicht von Bindegewebe gegenüber der Nervensubstanz (Fig. 1 and 2).

Nach van Gieson gefärbte Schnitte zeigen eine extreme Bindegewebswucherung zwischen den Nervenbündeln und Fibrillen. Axencylinder sind an mehreren Stellen garnicht zu finden. Die Anzahl der Bindegewebsmaschen, in welchen Axencylinder erhalten sind, verhält sich ungefähr zu denen, wo solche fehlen, wie 1:30-50.

Das Endoneurium scheint nicht verdickt zu sein, man hat mehr mit einer Neuritis parenchymatosa zu thun. Mit Carmin und Alaunhämatoxylin gefärbte Schnitte zeigen eine starke Verdickung der Membrana intima der Blutgefässe, die im Epineurium in etwas vermehrter Anzahl zerstreut sind. Ausserdem bieten die noch rorhandenen Axen- 
cylinder eine grosse Mannigfaitigkeit, indem die einen stark verdickt, die anderen sehr dünn erscheinen.

N. tibialis dexter nach Marchi. An Längs- und Querschnitten sind die Myelinscheiden ziemlich gut erhalten. Sie haben die Gestalt ziemlich homogener, gelblicher, leicht geschlängelter Streifchen, oder ziemlich regelmässiger gelblicher Kügelchen. Hie und da findet man in diesen Myelinscheiden und zwischen ihnen kleine schwarze Körnchen zerstreut, in einer vielleicht etwas übernormalen Anzahl. Grosse Klumpen von zerfallenem Myelin sind nicht zu sehen (Fig. 3 S. 184.)

Nach Weigert behandelte Präparate geben Bilder, die den vorigen

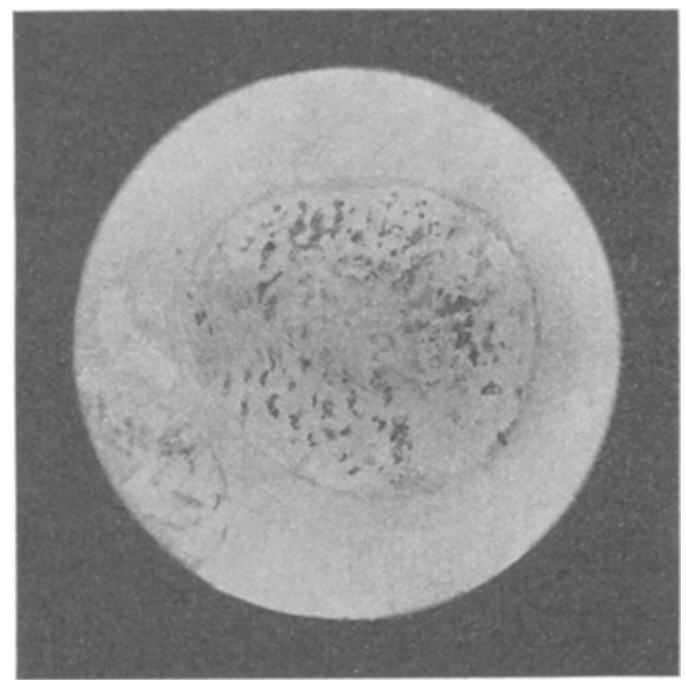

Fig. 1.

sehr ähnlich sind, $d . \mathrm{h}$. der Nerv erscheint fast ganz normal. Nur in den kleinen Bündeln sieht man besonders auf den Querschnitten kleine Felder zerstreut, wo schwarze Myelinklümpchen gänzlich fehlen.

Nach van Gieson gefärbte Schnitte zeigen ein normales Bild. Die Axencylinder sind überall unverändert erhalten. Sogar, wo nach den früheren Methoden das Myelin fehlt, sind die Axencylinder normal (Fig. 3).

Die Carminfärbung lieferte ein identisches Bild. Die Alaunhämatoxylinfärbung wies nirgends auf Kleinzelleninfiltration, noch auf eine Bindegewebskernwucherung. Nur die Wandungen kleiner Arterien waren im Verhältniss zu ihren Lumina etwas verdickt.

N. peroneus sinister nach Weigert. Die Veränderungen sind sehr bedeutend. Auf den Längsschnitten liegen die Myelinstreifen in einer gewissen Entfernung von einander. An sehr vielen Stellen sind die Streifen 
ausserordentlich dünn; an andָeren sieht man anstatt continuirlicher Fäden vereinzelte schwarze Klümpchen. Auf den Querschnitten sieht man in einzelnen Bündeln ganze Felder frei von Myelinscheiden. Hie und da sind kleine schwarze Körnchẻn and Klümpchen verschiedener Grösse zerstreut oder concentrische schwarze Streifchen.

Nach van Gieson. Die Veränderungen sind sehr stark. Axencylinder sieht man so gut wie keine. Das Endoneurium ist stark entwickelt: dicke, rothe Bindegewebszüge füllen fast das ganze Gesichtsfeld aus. Zwischen diesen dicken Bindegewebszügen sieht man auf den Querschnitten leere Maschen verschiedener Grösse zerstreut. Sehr selten sieht

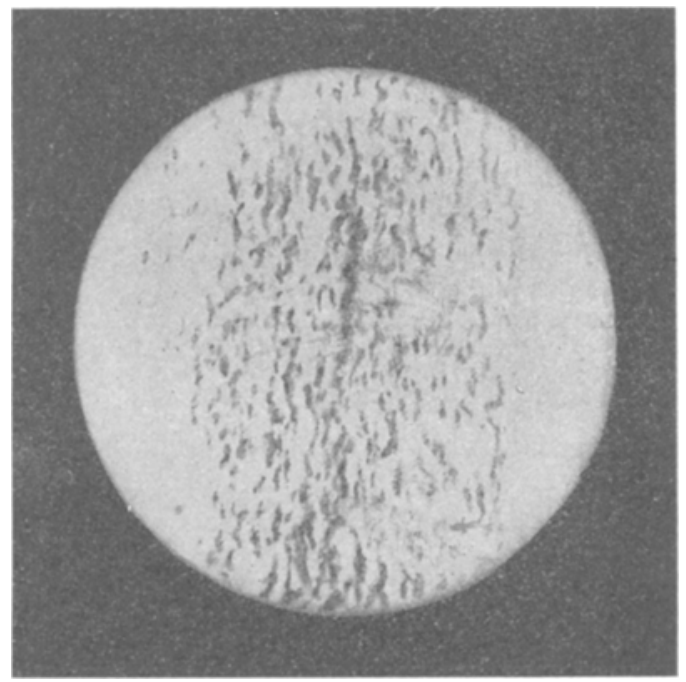

Fig. 2.

man in ihnen einen gewöhnlich stark verdünnten Axencylinder. ${ }_{\varepsilon}^{\text {DD }} \mathrm{Die}$ Carminfärbung zeigte gleichfalls sehr erhebliche ähnliche Veränderungen. Die Alaunhämatoxylinfärbung zeigte nirgends eine deutliche kleinzellige Infiltration. Die Gefässe zeigten keine deutlichen Veränderungen, abgesehen von einer geringen Verdickung der Wandungen.

Rückenmark mit Cauda equina. Querschnitte aus verschiedenen Höhen nach Marchi gefärbt.

Cauda equina am Ende des Conus terminalis.

Bei schwacher Vergrösserung (Ocul. 2, Obj. A A Zeiss $=1 \times 50$ ) sieht man ganze Felder entsprechend einzelnen Nervenwurzeln ganz hell, hie und da mit einzelnen darin zerstreuten Anhäufungen von gelbem Blutpigment. In einzelnen hellen Feldern,namentlich mehr vom Conus medullaris entfernt, sieht man dicht eingestreate kleine schwarze Körnchen, die dem Kohlenstaub ähnlich sind; in ganz vereinzelten gleichfalls peripher gelegenen 
Feldern sieht man gelbliche Streifen oder Kügelchen, wie sie an den mit Osmiumsäure behandelten Querschnitten der Nerven zu finden sind; dazwischen sind schwarze Klümpchen oder Körnchen verschiedener Configuration und Grösse zerstreut. Bei starker Vergrösserung findet man nach dieser Methode nur einzelne Rückenmarkswurzeln gefärbt, welche peripher liegen; dabei weisen sie starke Veränderungen auf; man sieht in ihnen eine Menge kleinerer und grösserer Körnchen, welche entweder in gelbliche Myelinscheiden eingeschlossen sind oder freiliegen.

Die Höhe des 5. Sacralsegments.

Im Rückenmark in den Hintersträngen in einem engen Saum zwischen

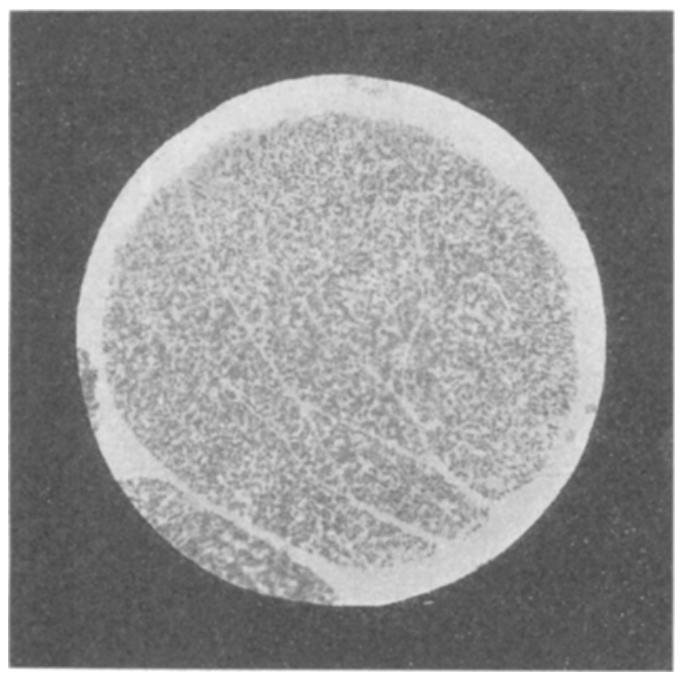

Fig. 3.

den Hinterhörnern sieht man an der Peripherie an Eingang der Hinterwurzeln difforme schwarze Myelinklümpehen ziemlich dicht zerstreut. Zahlreiche kleinere und grössere schwarze Körnchen findet man im ganzen Rückenmark, das intensiv weisslich aussieht und nur sehr wenige gewöhnlich gelbliche Scheiden enthält.

Was die Rückenmarksnervenwurzeln der Cauda equina anbetrifft, die dem Rückenmark anliegen, so sieht man hier wie im obigen Präparat die Mehrzahl der Wurzeln total entartet and ungefärbt: an einzelnen Stellen sieht man in der Umgebung dieser Felder zahIreiche schwarze Klümpchen und Körnchen. In den Wurzeln, die am meisten vom Rückenmark entfernt liegen und welche somit den oberen Lumbalsegmenten entspringen, sieht man hin und wieder die gelblichen Scheiden: Einzelne Bündel sind in ihnen gut erhalten, in anderen Bündeln derselben Wurzeln sieht man kaum noch schwarze Körnchen verschiedener Configuration auf dem hellen Hintergrunde zerstreut. 
Niveau des 5. Lumbalsegments.

Das Rückenmark zeigt auf dem Querschnitt erhebliche Veränderungen. Der ganze Querschnitt ist hell mit Ausnahme der Hinterstränge, die von einer Menge schwarzer Klümpchen durchsetzt sind, mit schwarzen Körnchen in der Mitte. Das sogenannte ventrale Hinterstrangsfeld enthält etwas weniger schwarze Degenerationsklümpchen. Ausserdem sind zahlreiche kleine schwarze Körnchen zwischen den Klümpchen zerstreut. Aehnliche kleine schwarze Körnchen findet man hie und da in den anderen Theilen des hellen Rückenmarks, aber ohne ausgesprochene Agglomeration. Längs der Hinterhörner laufen unterbrochene schwarze Streifchen, die aus Körnchen zusammengesetzt sind. Sie entsprechen den Hinterwurzeln, die auf dieser Höhe in das Rückenmark hineintreten. Die ventrolateralen Stränge zeigen einen mehr maschigen (areolären) Bau, was auf ältere Degenerationsveränderungen hinweist, so dass die Marchimethode hier nicht mehr die charakteristischen Bilder aufweisen konnte.

An den Rückenmarkswurzeln sind die Veränderungen ähnlich wie auf dem früheren Querschnitt. Die dem Rückenmark näher gelegenen Wurzeln, besonders die hinteren, zeigen leere helle Felder mit spärlichen kleinen Körnchen, die mitunter zwischen diesen Feldern angehäuft liegen. Die weit vom Rückenmark, besonders vorne und seitlich gelegenen Felder sind entweder ziemlich gut erhalten, oder zeigen Veränderungen, die auf einen frisch sich noch abspielenden Degenerationsvorgang hinweisen: Myelinklümpchen, die entweder homogen sind, oder verschieden configurirte Körnchen enthalten.

Niveau des 11 . Dorsalsegments.

Auf dem Querschnitt zeigen die ganzen Hinterstränge das Bild der Entartung. Zahlreiche Degenerationsklümpchen liegen zerstreut in dem ganzen Raum zwischen den Hinterhörnern bis zur Commissura posterior. Die Intensität der Degeneration ist überall gleichmässig. Auf den übrigen Feldern sieht man nur leicht zerstreute kleine schwarze Körnchen, ohne ausgesprochene Anhärfungen. In den Hinterhörnern ist nichts $A b$ normes zu finden.

Niveau des 5. Dorsalsegments.

Hier sind die Hinterstränge gleichfalls total und ohne Ausnahme degenerirt und zeigen im mikroskopischen Bilde zahlreiche Klümpchen und Körnchen verschiedener Grösse aus degenerirtem Myelin. Ausserdem sieht man an der Peripherie des Rückenmarks entsprechend den Kleinhirnbahnen und den Gowers'schen Strängen gleichfalls eine grössere Anzahl Myelinklumpchen und -Körnchen, als in den übrigen Theilen der Vertrolateralstränge.

\section{Methode Weigert-Pal (modificirt nach Wolters).}

1. Cauda equina am Ende des Conus medullaris (Fig.4 S. 186). Die Mehrzahl der Felder, die einzelnen Rückenmarksnerrenwurzeln entsprechen, ist hell, ohne irgend welche Spur von Färbung. Sehr spärliche, mehr vom Conus entfernte Wurzeln sind ziemlich gut gefärbt, die meisten weisen Veränderungen im Myelin auf: die schwarzen Myelinklümpchen sind seltener und von farblosen Feldern von einander getrennt. An den im Längsschnitt getroffenen Wurzeln sieht man an vielen Stellen auf einem hellen Hinter- 
grunde schmale rosenkranzähnliche, oft unterbrochene Streifchen, die keine compacte Masse bilden, sondern frei von einander in Abständen liegen.

2. Niveau des 5. Sacralsegments.

Die weisse Substanz des ganzen Querschnitts färbt sich nach dieser Methode schwach. Bei starker Vergrösserung sieht man spärlich zerstreute Myelinstreifchen in der grauen Substanz und Myelinkreischen in der weissen. Dünne Myelinfäden zeigen hie und da eine Verdickung. Die feinen Körnchen sind zahlreicher als die gröberen Klümpchen. Einzelne Nervenwurzeln, vorne und seitwärts, weiter vom Rückenmark entfernt, sind gut gefärbt; alle hinteren und die, welche näher zum Rückenmark liegen, sind entweder ganz farblos oder enthalten nur hie und da schwarze Pünktchen. In einigen Wurzeln sieht man, wie an den nach Marchi gefärbten Präparaten, kleine umschriebene Felder totaler Degeneration neben anderen, die ganz gut erhalten sind, liegen.

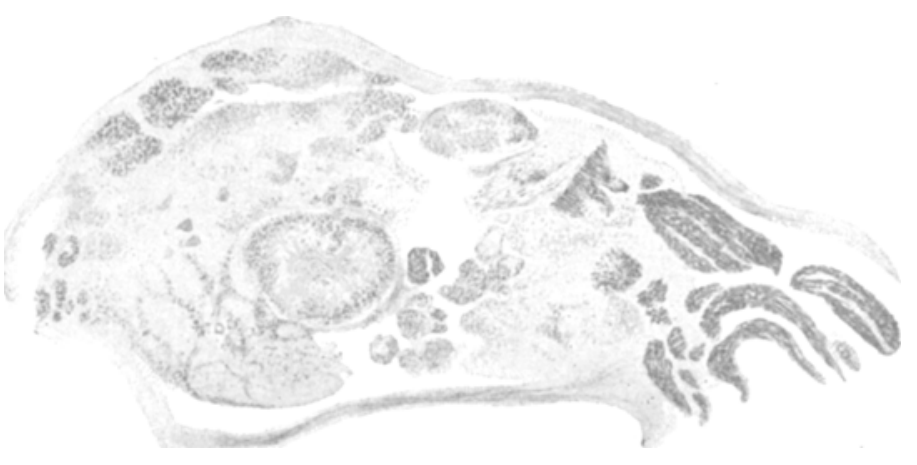

Fig. 4.

\section{Niveau des 5. Lendensegments.}

Hier ist gleichfalls der ganze Querschnitt blässlich. Die Ventrolateralstränge sind schwach gefärbt; in einigen Stellen des linken ventrolateralen Stranges sieht man mehrere, ganz farblose, d. h. vollständig myelinfreie Herde. Die graue Substanz sieht bei schwacher Vergrösserung ganz blass aus; bei einer stärkeren Vergrösserung findet man in ihr zahlreiche verdünnte Myelinstreifchen. Die stark degenerirten Hinterstränge sind nur äusserst schwach gefärbt; etwas mehr schwarze Punkte sieht man in der vorderen Partie der Hinterstränge (ventrales Hinterstrangfeld). Von den Rückenmarksnervenwurzeln sind nur einzelne theilweise leidlich gefärbt: einige vorne und 2-3 auf den Seiten, die weiter vom Rückenmark liegen. Die übrigen sind ganz ungefärbt geblieben und enthalten blos hie and da an der Peripherie vereinzelte schwarze Pünktchen.

4. Niveau des 11. Dorsalsegments.

Auf dem Querschnitt erscheinen die Hinterstränge auffallend schwach gefärbt. In der Gegend der Commissura posterior ist die Färbung etwas deutlicher. In den übrigen Theilen sieht man keine deutlichen Veränderungen, abgesehen von einer vermehrten Blässe der grauen Substanz. 
5. Niveau des 5. Dorsalsegments.

Hier fällt auch eine bedeutende Blässe der Hinterstänge auf; das ventrale Hinterstrangfeld ist etwas besser tingirt. Die Clarke'schen Säulen sind etwas blasser als normal (Fig. 5).

Färbung nach van Gieson mit Carmin und Nachfärbung mit Alaunhämatoxylin.

Cauda equina am Ende des Conus medullaris.

Sehr erhebliche Veränderungen. Viele Rückenmarksnervenwurzeln, besonders die, welche in der Mitte liegen, bilden compacte, intensiv rosa gefärbte Bindegewebsbündel. Stärker gefärbte kreisförmige Streifen lassen die einzelnen Wurzeln von einander unterscheiden. In anderen wieder sieht man rothe Bindégewebsfelder innerhalb relativ weniger degenerirter

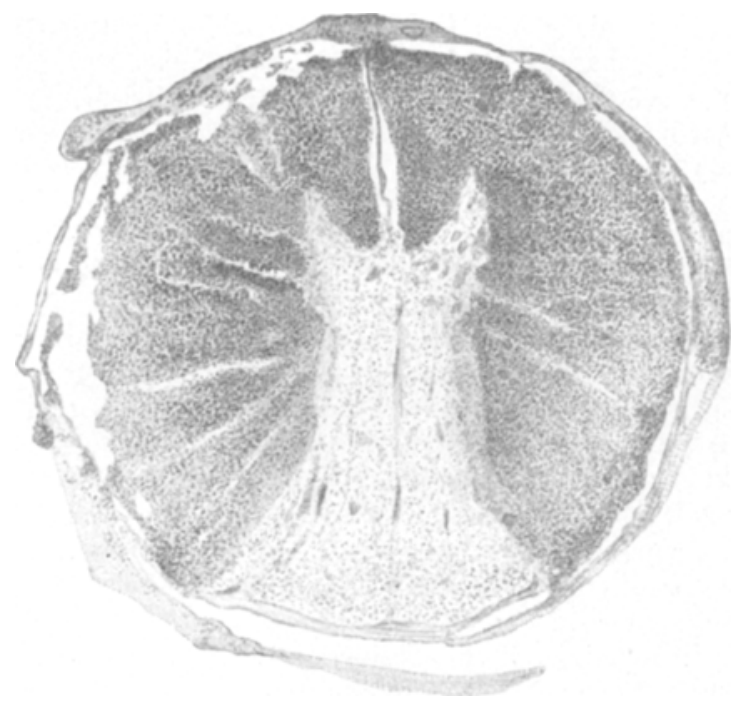

Fig. 5 .

Wurzeln; es sind dieselben, welche, nach den früheren Methoden gefärbt, noch Reste von verändertem Myelin zeigten; wieder in anderen mehr central gelegenen Wurzeln finden wir ein grob- oder feinmaschiges Netz. Axencylinder lassen sich aber nirgends entdecken. Zwischen den Wurzeln, welche an vielen Stellen zu einer compacten Bindegewebsmasse zusammengewachsen sind, sieht man zahlreiche Gefässe 'mit verdickten Wandungen und perivasculärer kleinzelliger Infiltration. In einzelnen Arteriolen ist die Intima verdickt, das Endothel kreisförmig abgehoben, und in seinem Lumen sowie zwischen ihm und der Gefässwand sieht man rothe Blutkörperchen. In einigen kleinen Gefässen sind die Thromben hyalin degenerirt. Die Dura mater ist überall stark verdickt, entweder liegt sie frei auf, so dass das Spatium subdurale erhalten ist, oder sie ist durch Bindegewebszüge fest mit den degenerirten Nervenwurzeln verwachsen. 
Zwischen einzelnen Nervenwurzeln, welche eigentlich in Bindegewebszüge verwandelt sind, und in ihrem Innern sieht man rothgefärbte, ziemlich scharf umschriebene Felder von verschiedener Configuration. Wenn ihre Umgebung eine starke kleinzellige Infiltration zeigt, färben sich die Kerne in den Feldern entweder nur sehr blass oder garnicht; in einzelnen Feldern sieht man das Lumen kleiner Gefässe mit gänzlich undifferenzirter Wandung, welche unmittelbar in das umgebende compacte Gewebe übergehen. Diese Felder machen den Eindruck von einem gummösen Granulationsgewebe mit Verkäsungsherden und Bindegewebsnarben. Die Zahl der im ganzen Gesichtsfelde zertreuten Gefässe ist stark vergrössert.

Niveau des 5. Sacralsegments.

Im Rückenmark selbst sowie in den es umgebenden Wurzeln findet man sehr erhebliche Veränderungen. Das Rückenmark selbst färbt sich mit Carmin und nach ran Gieson äusserst schlecht. Die graue Substanz hebt sich fast gar nicht von der weissen ab. Es lassen sich nur die Hinterhörner einigermassen unterscheiden. Somit sieht man nur auf dem blassröthlichen von zahlreichen Maschen durchsetzten Hintergrunde, was auf den Zerfall der Rückenmarkssubstanz hinweist, eine Menge kleiner, vorwiegend capillärer Gefässe, deren Mehrzahl mit Blut angefüllt ist. Es ist schwer zu unterscheiden, ob diese zahlreichen breiten Capillaren aus einer Erweiterung alter Gefässe hervorgegangen, oder ob sie neu gebildet sind. An vielen Stellen des Gesichtsfeldes liegen rothe Blutkörperchen frei. Auf dem ganzen Querschnitt des Rückenmarks ist kein einziger Axencylinder zu finden. In der grauen Substanz ist gleichfalls keine einzige Nervenzelle zu sehen. An einigen Stellen an der Peripherie des Rückenmarks, besonders in der Gegend des rechten Hinterhorns und des rechten ventrolateralen Stranges sieht man intensiver roth gefärbte Felder. Die Gesammtanzahl der Neurogliakerne ist etwas vermehrt, man findet aber keine Kleinzellenanhäufung in der Marksubstanz.

Die Nervenwurzeln zeigen sehr erhebliche Veränderungen, so wie auf dem obigen Präparat. In den näher zum Rückenmark, besonders hinten und an den Seiten gelegenen Bündeln lassen sich einzelne Wurzeln nicht unterscheiden. An einigen Stellen bildet die Peripherie des Rückenmarks mit der Pia mater, den Nervenwurzeln und sogar der Dura eine homogene Gewebsmasse, die von kleinen Zellen stark infiltrirt ist, ohne eine Spur von Axencylinder, hie und da mit erhaltenen Maschen und zahlreichen stark veränderten Blutgefässen; an vielen Stellen sieht man reichliche Anhäufungen von kleinen Zellen und kleinere und grössere Blutextravasate. In den nach vorne liegenden und mehr vom Rückenmark entfernten Nervenwurzeln findet man an einigen Stellen besser, an anderen wieder weniger gut erhaltene Axencylinder. Die einen sind stark verdickt, intensiv tingirt, andere - in den benachbarten Maschen - sehr dünn, andere wieder sehr blass. In den Bindegewebsscheiden des Perineurium sieht man hie und da kleinzellige Infiltrate, die tief in das Innere der Nervenwurzeln sich erstrecken. Die Art. spinalis anterior ist verdickt und in ihrem Lumen verengert; die Membrana elastica ist nur schwer zu finden; auf einer Seite ist im lymphatischen perivasculären Raum eine Anhäufung kleiner Zellen zu sehen. Die Vena spinalis anterior zeigt keine deutlichen Veränderungen. 
An 2 Stellen - vorne und anf der Seite - sieht man: mittelgrosse, intensiv roth tingirte Felder mit dem Charakter eines gummösen Gewebes, das käsig degenerirt und in eine Biedegewebsnarbe verwandelt ist; dieses Feld erinnert an eine ähnliche Stelle, die im obigen Querschnitt beschrieben war.

\section{Niveau des 3. Sacralsegments.}

In den mit Alaunhämatoxylin gefärbten Präparaten sieht man zahlreiche kleine Zellenagglomerate rings um die Gefässe herum in den Lymphränmen der Membrana externa, zwischen den Nervenwurzeln rings um die Arteria spinalis anterior herum, in der verdickten Pia mater, wo der Sulcus longitudinalis anterior beginnt. Die Zahl der stark veränderten Gefässe ist bedeutend vermehrt. In einigen Nervenwurzeln an entarteten, mitunter die Hälfte des Querschnittes der Wurzel einnehmenden Stellen sieht man scharf abgegrenzte Felder mit ziemlich gut erhaltenen Axencylindern und einer regulären Anzahl der Kerne.

Die Marksubstanz ist stark verändert, dünn und maschig. Zahlreiche Blutextravasate und Capillarenramificationen. Nervenzellen und Axencylinder sind nicht zu sehen.

Die oben beschriebenen verkästen Gummata sind weder zwischen den Rückenmarkshänten, noch zwischen den Nervenwurzeln zu finden.

Niveau des 5. Lumbalsegments.

Das Rückenmark zeigt auf dem Querschnitt etwas leichtere Veränderungen, als auf der obigen Höhe. Die Allgemeinstructur ist etwas besser erhalten und die grawe Substanz hebt sich deutlicher von der weissen ab, obgleich das ganze Aussehen des Rückenmarks und eine maschige Structur auf den Zerfall des Gewebes hinweist. In den Vorderhörnern der grauen Substanz sieht man die motorischen Zellen stark verändert: sie sind durchweg ganz homogen gefärbt oder in eine körnige Masse verwandelt, haben eine mehr kugelige Gestalt angenommen; bei den meisten sieht man weder die protoplasmatischen, noch die Axencylinderfortsätze; die pericellulären Räume sind stark erweitert. Kerne lassen sich nur in einzelnen Zellen finden: sie liegen dann meistens peripher. Die Blutcapillaren sind zahlreich auch in der weissen Marlssubstanz, besonders aber in den Vorderhörnern der grauen Substanz vorhanden.

Die weisse Substanz ist stark verändert. Die Axencylinder treten hie und da als rothe Pünktchen in schwach gefärbten gelblichen Myelinscheiden hervor. Vorwiegend findet man aber Lü̈cken und ziemlich weite Maschen in allen Strängen der weissen Substanz. Kleinzellenagglomerate sind in dem Rückenmark nicht zu finden. In den das Rückenmark umgebenden Nervenwurzeln sind die Veränderungen dieselben wie an den früheren Präparaten: Bindegewebsdegeneration vieler Bündeln (rothe Streifen), Zusammenwachsen vieler Wurzeln mit einander und mit der Pia und Dura mater zu einer einzigen Masse, in der hie und da zahlreiche Kleinzelleninfiltrate hervortreten. Nur an der extremen Peripherie des Präparates sieht man ziemlich gut erhaltene Axencylinder und einen nahezu normalen Bau der Nervenwurzeln.

Im Bereich der Vorderwurzeln sieht man beiderseits zwei charakteristische submiliare Felder; sie färben sich mit Carmin intensiv roth; bei starker Vergrösserung sind sie aus mehreren vereinigten Feldern zusammen- 
gesetzt, die aus dünnen leicht geschlängelten, nur lose an einder anliegenden rothen Streifen zusammengesetzt sind; inmitten einiger dieser Felder sieht man ein Gefässlumen, dessen Wände stark verändert sind und ihren Bau vollkommen eingebüsst haben. Diese kreisförmigen Streifen lagern sich concentrisch vorwiegend um das Lumen dieser Gefässe herum. Das Alaunhämatoxylin lässt an diesen ganzen Feldern gar keine Neurogliakerne entdecken. Hie und da sieht man zwischen diesen rothen Streifen stark veränderte Axencylinder entweder als grosse, intensiv rothe Klümpchen, oder als kleine blassrothe Pünktchen hervortreten. Stark geschwollene Axencylinder liegen an vielen Stellen neben anderen, welche im Verschwinden

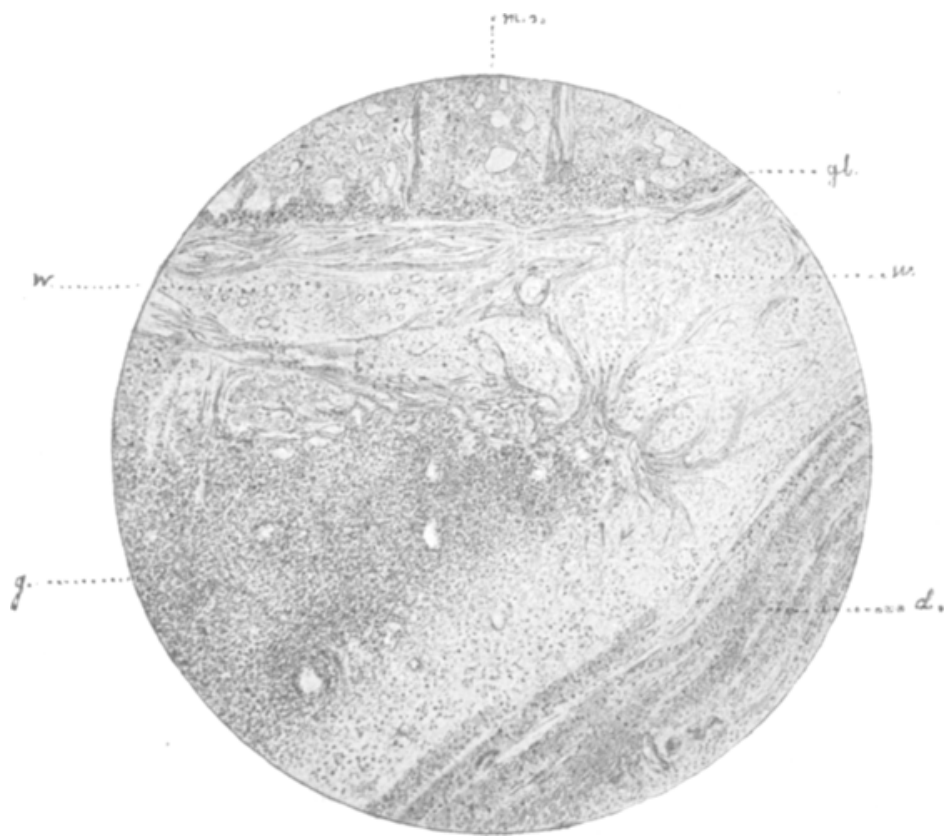

Fig. 6 .

begriffen sind. Diese Felder bestehen zweifelsohne aus gummösem käsig degenerirten Gewebe mit Umwandlung in Bindegewebe (siehe Schmaus, Pathologische Anatomie des Rückenmarks. - Wiesbaden 1901. Fig. 162); sie gehen ziemlich scharf über in ein maschiges Gewebe mit zahlreichen Kleinzelleninfiltrationen (Fig. 6).

Die Art. spinalis anterior hat eine deutlich verdickte Intima, deren Dicke zweimal übertrifft die der Museularis und der Externa zusammen. Die Membrana elastica ist nicht überall erkennbar. Kleinzellige Infiltrate sind in der Intima nicht zu sehen, es sind vielmehr einzelne runde oder längliche Kerne. Die Venen zeigen geringere Veränderungen, obgleich zum Beispiel die Spalten der Intima der Vena spinalis 
Syphilis des Rückenmarks u. seiner Häute in d. Lumbosacralgegend. 191

anterior ziemlich gross sind. An einzelnen stark verdickten kleinen Gefässen sind Vasa vasorum zu sehen.

Niveau des 3. Lumbalsegments.

Die Veränderungen in der Marksubstanz sind viel geringer. Der Bau des Rückenmarks beginnt auf dem Querschnitt sich der Norm zu nähern. Die Nervenzellen der Vorderhörner der grauen Substanz sind in allen Gruppen wenig verändert. Die von ihnen in die Vorderhörner auslaufenden Fortsätze sind ziemlich gut erhalten; die Axencylinder der ventrolateralen Stränge gleichfalls. Hie und da sieht man grössere Maschen frei von Axencylindern. In den Hintersträngen ist die Neuroglia und das Bindegewebe stark hypertrophisch. Die Neurogliaschicht an der Peripherie des Rückenmarks, die sogenannte Gliaschicht, ist etwas verdickt. Die Pia mater ist in den hinteren Partien leicht verdickt und stellenweise kleinzellig infiltrirt. Die Vorderwurzeln, sogar die dem Rückenmark dicht anliegenden, sind normal. Die Hinterwurzeln, namentlich links, sind noch degenerirt, aber nicht so stark wie auf den früheren Querschnitten. Ja sogar in den dicht dem Rückenmark anliegenden Hinterwurzeln findet man hie und da schwarz gefärbte Axencylinder.

Niveau des 11. Dorsalsegments.

Abgesehen von der Wucherung der Neuroglia and des Bindegewebes in den Hintersträngen sieht das Rückenmark auf dem Querschnitt fast normal aus. Die rothe Färbung der Hinterstränge ist hier intensiver, als auf dem früheren Querschnitt. Sonst ist im Rückenmark nichts Besonderes $z u$ sehen. Auffallend ist nur die ausgeprägte Degeneration der rechten hinteren Nervenwurzel, wo man eine starke Bindegewebswucherung findet. Die Wurzel ist dicht verwachsen mit der verdickten Pia mater, welche an dieser Stelle leicht kleinzellig infiltrirt ist. Die linke Hinterwurzel und beide Vorderwurzeln sind ähnlich, aber weniger degenerirt und zeigen auch keine deutliche Infiltration. Einzelne Bündel der Vorderwurzel zeigen eine vermehrte Anzahl Bindegewebszüge mit entsp rechenden specifischen Veränderungen.

Niveau des 5. Dorsalsegments.

Das Rückenmark zeigt keine Veränderungen, abgesehen von der oben geschilderten Degeneration der Hinterstränge; man sieht dabei die Wucherung der Neuroglia und des Bindegewebes hauptsächlich von den Bindegewebssepta ausgehen, in welchen die Blutgefässe in das Rückenmark hineintreten. Die Hinterwurzeln haben mehr Bindegewebsstreifen, welche ausserdem noch etwas dicker sind. Die Vorderwurzeln zeigen ähnliche Veränderungen in geringerem Grade. An der Peripherie, entsprechend der Gegend der Kleinhirnbahnen und der Gowers'schen Stränge, findet man Veränderungen, die für secundäre Degeneration charakteristisch sind: netzförmige Structur des Gewebes, fehlende oder degenerirte Axencylinder, Wucherung der Neuroglia, gequollene Myelinscheiden. In den Ventrolateralsträngen ist die Zahl der Gefässe vermehrt.

Wenn wir nun in knappen Worten das klinische und das spathologisch-anatomische Bild des Falles zusammenfassen wollen, so stellt es sich folgendermassen heraus: 
Die Kranke, 20 Jahre alt, kam in das Krankenhaus mit Klagen über allgemeine Schwäche, besonders der Beine, und über Schmerzen im ganzen Körper. Die Anamnese hat Abusus in Baccho, aber keine luetische Infection festgestellt. Die objective Untersuchung ergab: Abnahme der motorischen Kraft der Beine, in mässigem Grade auch der Arme, Druckempfindlichkeit der Nervenstämme, disseminirte und veränderliche Sensibilitätsstörungen, fehlende Patellar - und Achillessehnenreflexe, dabei hysterischer Seelenzustand und Stigmata. Nach. 2 Monaten Krankenhausaufenthalt trat die Incontinentia urinae auf, bald darauf Decubitusgeschwüre der Hinterbacken und trophische Störungen in Gestalt runder, tief bis zum Knochen dringender Geschwüre an mehreren Stellen der Vorder- und Seitenflächen der unteren Extremitäten. Tod in Folge septischer Infection und einer hypostatischen Pneumonie.

Die Obduction ergab eine hypostatische Pneumonie als die endgültige Todesursache, sonst Veränderungen vorwiegend im Nervensystem, und zwar ausgesprochene Veränderungen vorwiegend parenchymatösen Charakters in den $\mathrm{Nn}$. tibialis und peroneus sinistri neben fast vollkommen unverändert gebliebenem N. peroneus dexter. Die Veränderungen im Rückenmark waren hauptsächlich im Lumbal, und Sacralabschnitt localisirt. In der Gegend des Conus medullaris waren sämmtlicke Nervenwurzeln total degenerirt mit Ausnahme der peripheren, welche somit aus den oberen Lumbalsegmenten entspringen.

Zwischen allen drei Rückenmarkshäuten kleinzellige Infiltration, hier und da miliare oder verkäste Gummata. Die Rückenmarkssubstanz ist verdünnt und gelockert, von netzartigem Bau mit stark veränderten Axencylindern, stellenweise kleinzellig infiltrirt, die Conturen der grauen Substanz sind verwaschen. Je höher man kopfwärts steigt, desto geringer werden die Veränderungen der Häute, der Marksubstanz und namentlich der Gefässe. Im Dorsalabschnitt treten hervor secundäre aufsteigende Degenerationen der Hinterstränge, der Kleinhirnbahnen, der Gowers'schen Stränge. Die Veränderungen der Rückenmarkshüllen sind auf der Höhe der mittleren Dorsalsegmente äusserst gering. Das Gehirn zeigte makroskopisch keine Veränderungen.

Wir hatten es somit mit einem Fall von Syphilis zu thun, der in den unteren Rückenmarksabschnitten localisirt war.

Die Schwierigkeiten der Diagnose waren vor allen Dingen durch eine schwere Hysterie bedingt, welche die richtige Abschätzung der einzelnen Erscheinungen störte. Weiter liessen die starke Druckempfindlichkeit der Nervenstämme, die fehlenden Patellar- und Achillessehnenreflexe, das Schmerzgefühl in allen Extremitäten, der bedeutende anamnestisch festgestellte Abusus in Baccho an eine diffuse 
Polyneuritis denken. Die Abnahme der Kräfte in allen Extremitäten, besonders in den Beinen, ohne deutliches Befallensein einzelner Muskelgruppen wurde zum Theil mit der Erkrankung der Nerven, zum Theil mit der Hysterie in Verbindung gesetzt. Der Gang der Kranken erinnerte thatsächlich an die hysterische Abasie (Zusammenknicken der Beine). Eine Erkrankung des Rückenmarks, an die man denken konnte wegen der fehlenden tiefen Reflexe in den unteren Extremitäten, wurde anfänglich nicht angenommen, da keine Blasenstörung und keine constanten Sensibilitätsstörungen der Beine vorhanden waren. Zwar konnte die allgemeine Schmerzhaftigkeit des Körpers ein Zeichen der al]gemeinen Reizerscheinung der Rückenmarkshäute sein, sogenannte Irritatio meningealis und als solche als ein Frühsymptom der Syphilis des Nervensystems gelten, aber beim Vorbandensein schwerer hysterischer Symptome (die Kranke hatte ab. und $\mathrm{zu}$ hysterische Anfälle) haben wir ihr nur einen functionellen Charakter zugeschrieben. Die Möglichkeit der Syphilis bei einer jungen Person von verdächtiger Führung liess sich nicht in Abrede stellen, trotzdem dass diesbezügliche anamnestische Daten fehlten. Es ist ja bekannt, wie schwer solche Daten bei Frauen zu erlangen sind. Fournier sagt anlässlich der Syphilis insontium (d. h. solcher Franen, die gar keine Ahnung von ihrer Infection haben), dass er bei 50 Proc. der Frauen mit tertiären Lueserscheinungen in der Anamnese gar keine Andeutung auf die erfolgte Infection finden konnte.

Das Fehlen der tiefen Reflexe der unteren Extremitäten und die disseminirten Schmerzen liessen noch an Tabes denken, aber gegen eine solche Annahme sprachen die gut reagirenden Pupillen, das Fehlen von Sensibilitätsstörungen sowie von anderen Erscheinungen, die charakteristisch für dieses Leiden sind, ebenso, wenn schon nicht unbedingt, das jugendliche Alter der Kranken.

Nach 3 Monaten Hospitalaufenthalt trat die Incontinenz der Blase auf. Anfänglich wurde diese Erscheinung für eine functionelle Sphincterschwäche gehalten; die Kranke lag neben einer Tabeskranken, die beständig den Harn unter sich laufen liess. Nach 8 Tagen traten auf der vom Harn fortwährend macerirten Haut der Hinterbacken rasch wachsende Decubitusgeschwüre auf, und es zeigten sich, wie das oben ausführlich beschrieben ist, auf den Vorder- und Seitenflächen der unteren Extremitäten an fast symmetrischen Stellen anfangs umschriebene Röthungen, später Bläschen mit leicht getrübtem serösem Inhalt und schliesslich in die Tiefe dringende Geschwüre mit scharf abgeschnittenen Rändern, ohne Röthung in der Peripherie.

Die sich rasch entwickelnden breiten und tiefen DecubitusDeutsche Zeitschr, f. Nervenheilkunde. XXIV. Bd. 
geschwüre der Hinterbacken machten den Eindruck des sogenannten Decubitus acutus (échare à développement rapide - Chareot) Derartige trophoneurotische Hautaffectionen pflegen, wie bekannt; ebenso gut aufzutreten bei Erkrankungen des Gehirns (besonders bei Blutergüssen, Erweichungen - Monakow) wie des Rückenmarks (Myelitis, Trauma des Rückenmarks) und der peripheren Nerven. Die Pathogenese des Decubitus ist, trotz vieler diesbezüglichen Untersuchungen, noch nicht aufgeklärt. Was ihr Entstehen anbetrifft, so steht es aber fest, dass sie am öftesten beim Leiden der unteren Rückenmarksabschnitte vorkommen.

Goldflam (l. c.) hat in seinem 8. Fall, wo er einen grossen Erweichungsherd gefunden hatte in den vorderen Strängen und Höruern auf der Höhe des 3. und 4. Lumbalsegments, intra vitam ausgiebige Decubitusgeschwüre beobachtet, mit ausgefressenen, unterminirten Rändern in der Gegend des Kreuzbeins und der Trochanteren sowie auf den Fersen und an den Malleoli externi. Im 11. Fall (Myelitis dorso-Iumbalis acuta) notirte Goldflam neben Decubitusgeschwüren der Hinterbacken auf der Innenseite der Kniee anfangs umschriebene rothe Flecken und dann Blasen mit einem serösen Inhalt. Der Kranke starb unter pyämischen Erscheinungen am 18. Tage des Hospitalaufenthaltes und am 32. Krankheitstage, 5 Monate nach der primären Inficirung. Grosse Decubitusgeschwüre bei Erkrankungen der unteren Rückenmarksabschnitte erwähnen auch Orłowski (l. c. Fall I) und Lamy ${ }^{1}$ ) (Fall XIII).

Sogar tiefe und ausgiebige Decubitusgeschwüre sind bei Weitem keine Seltenheit. In unserem Falle hatten wir aber noch zu thun mit ungewöhulichen trophischen Störungen in der Gestalt von tiefer Nekrose an Stellen, die keinem Druck weder des Bettes, noch der anderen Extremität ausgesetzt sind.

Leyden und Goldscheider ${ }^{2}$ ) bemerken, dass die Myelitis lumbosacralis eine schnelle Ausbildung schwerer trophischer Störungen an den unteren Extremitäten und an den Hinterbacken verursacht: es entstehen Decubitusgeschwüre, Blasen, Oedeme (S. 388).

Flatau und Koelichen ${ }^{3}$ ) schreiben in der Arbeit „Ueber die Rückenmarksentzündung": „Ausser den eigentlicheu Decubitusge-

1) Lamy, De la méningo-myélite syphilitique. Nouvelle Iconographie de la Salpêtrière. 1893.

2) Leyden und Goldscheider, Die Erkrankungen des Rückenmarks und der Medulla oblongata (bei Nothnagel). Wien 1865.

3) Flatau und Koelichen, O zapaleniu rdzenia. (Ueber die Myelitis.) Warschau 1902. 
schwïren finden wir oft auf der Haut an Stellen, wo die gelähmten Extremitäten sich gegenseitig berühren, Blasen mit seröser Flüssigkeit. Nach einiger Zeit platzen sie und hinterlassen lange nicht zuheilende Geschwüre. In seltenen Fällen treten auf der Haut verschiedene Eruptionen hervor, manchmal in Gestalt eines Pemphigus oder Herpes zoster" (S. 78).

Die trophischen Störungen, die auf nerrösem Grunde entstehen, behandelt ausführlich Cassirer') in einer speciellen Monographie. Aus den einzelnen Kapiteln dieser Arbeit und der Beschreibung der verschiedenen Erkrankungsformen, die übrigens etwas künstlich eingetheilt sind, ist ersichtlich, dass die bei unserer Kranken aufgetretenen trophischen Störungen nur schwer ausschliesslich auf den sogenannten Decubitus acutus zurückzuführen wären. Jene umgrenzten Brandherde, die ziemlich symmetrisch auf den Vorder- und Seitenflächen der unteren Extremitäten und sogar auf dem Bauch zerstreut waren, sollte man viel eher zur Kategorie der circumscripten disseminirten Hautgangrän, der multiplen Hautgangrän oder der multiplen, neurotischen Hautgangrän, wie sie Andere nennen, rechnen. Der Brand' war vorwiegend trocken. Es ging ihm voraus eine begrenzte Röthung der Haut und später eine Blase mit serösem Inhalt. Ein ähnliches Leiden, das man vorwiegend bei Hysterischen findet, nennt Kaposi „Herpes zoster gangraenosus hystericus". Cassirer schreibt (1. c. S. 546), dass die oben genannte disseminirte Hautgangrän auch bei organischen Erkrankungen auftreten kann (Gliosis spinalis, Neuritis ascendens, Herpes zoster). Bei unserer Patientin waren die beiden Bedingungen rorhanden, welche das Entstehen der disseminirten Hautgangrän begünstigen.

Erstens hatte sie neben einer verhältnissmässig geringen Zerstörung der Rückenmarksubstanz im lumbosacralen Abschnitt eine fast totale Degeneration sämmtlicher Nervenwurzeln der Cauda equina, in Folge einer heftigen Afficirung der Rückenmarkshäute. Dass dabei auch fast alle vasomotorischen und trophischen Fasern (letztere sind freilich heute noch hypothetisch) untergehen mussten, lässt sich nicht bezweifeln. Samuel (citirt von Cassirer S. 88) führt in seiner Arbeit "Die trophischen Nerven" einen Fall Remak's an, wo sich ein Decubitus gangraenosus ausgebildet hatte in Folge des Druckes auf die Cauda equina. Charcot citirt einen Fall ron Decubitus gangraenosus in Folge des Druckes einer von dem Becken ausgegangenen Geschwulst auf die $\mathrm{Nn}$. ischiadicus und cruralis.

1) R. Cassirer, Die vasomotorisch-trophischen Neurosen. Berlin 1901. 
Zweitens war unsere Patientin eine ausgesprochene Hysterica. Gilles de la Tourette hält die hysterische Gangrän für den höchsten Grad der vasomotorischen Diathese. Er sagt: „Au premier degrès du processus c'est la dermographie, au deuxième l'oedème, puis la lésion vésiculeuse, bulleuse ou pustuleuse, à un degrès ultime se montre la gangrène" (Cassirer S. 541).

In unserem Falle genügten die Bedingungen vollkommen zum Entstehen der Gangrän, obgleich das klinische Bild zu den seltenen gehörte.

Diese Seltenheit bestand in der Localisation des syphilitischen Vorgangs in den untersten Abschnitten des Rückenmarks, welche gar nicht oft vorkommt. Die syphilitische Erkrankung des Conus medullaris und der Cauda equina mit einer mikroskopisehen Untersuchung ist von Eisenlohr und Westphal (citirt bei Orłowski) beschrieben worden; Orłowski bat auch 2 klinische Fälle beschrieben (Fall 27 und 28). Der gewöhnliche Sitz der syphilitischen Erkrankung des Rückenmarks ist der dorsale Abschnitt, was mit den Besonderheiten der Vascularisation des Rückenmarks zusammenhängt; die Rückenmarksubstanz wird nämlich versorgt durch 2 Blutgefässsysteme: das eine, die sogenannte Vasocorona, setzt sich aus Gefässen zusammen, welche rechtwinklig von der Peripherie in die Tiefe des Rückenmarks als Endarterien hineindringen, das andere ist das System der Arteria spinalis anterior. Im Dorsalabschnitt sind die secundären und Seitenäste äusserst spärlich, wie das Adamkiewicz und Kadyi nachgewiesen haben; allerlei Circulationsstörungen, die meistens durch Druck der infiltrirten und verdickten Pia mater auf die Gefässe entstehen, werden nur mit grossen Schwierigkeiten ausgeglichen, so dass es leicht zur Erweichung der Rückenmarkssubstanz kommen kann. In der Lumbal- und Cervicalgegend sind die Seitenäste zahlreicher und derartige Vorgänge kommen viel seltener vor. Die syphilitischen Lähmungen der unteren Extremitäten sind deswegen gewöhnlich spastisch und nur selten dauernd schlaff.

Die auf unseren Präparaten gefundenen pathologisch-anatomischen Veränderungen sind ebenfalls bemerkenswerth. Wie bekannt, sieht die Mehrzahl der Forscher in Uebereinstimmung mit Julliard die Syphilis an als ein Leiden vorwiegend des. Lymphgefässsystems, besonders jener lymphatischen Räume, welche so zahlreich auch unter den Hirnhäuten vertreten sind. Oppenheim hält die Meningitis cerebrospinalis für die häufigste und die bestcharakterisirte Form der Syphilis des Nervensystems. Unser Fall bestätigt diese Annahme, da hier thatsächlich die Häute den Ausgangspunkt des specifischen Vorgangs darstellten. Wir haben ja vor allen Dingen eine Ver- 
dickung der Dura in den unteren Rückenmarksabschnitten gefunden; es war eine Art Pachymeningitis, wie wir sie öfter in dem Cervicalabschnitt finden. Die Dura sowie die Pia und Arachnoidea - sie waren alle verdickt und kleinzellig infiltrirt, die Nervenwurzeln waren an vielen Stellen förmlich von gewuchertem Bindegewebe umwachsen; wo die Nervenwurzeln noch keine Zeit gehabt haben, sich in Bindegewebsstränge zu verwandeln, sah man deutlich die Infiltration längs der Bindegewebssepta in das Innere der Wurzel hineindringen. Die verdickten und veränderten Rückenmarkshäute drückten auf die Gefässe und beeinträchtigten die Ernährung der Marksubstanz, aber ohne es zur Ausbildung grosser Erweichungsherde kommen zu lassen. Der Ausdruck dieses Zustandes war der maschige (alveoläre) Bau der Rückenmarkssubstanz. Das Fehlen grösserer Herde lässt sich dadurch erklären, dass man jedenfalls, namentlich in den auf der Vorderseite des Rückenmarks verlaufenden Gefässen keine stärkeren Veränderungen finden konnte.

Die Mehrzahl der Forseher, die sich mit der Syphilis des Nervensystems beschäftigten, sind dartiber einig, dass bei der Gehirnsyphilis sich die specifischen Veränderungen vorwiegend iu den Arterien, bei der Rückenmarkssyphilis aber in den Venen concentriren. Den Charakter dieser Veränderung habe ich schon früher Gelegenheit gehabt an einem anderen Orte zu besprechen. ${ }^{1}$ ) Hier will ich nur bemerken, dass in meinem Fall vorwiegend die kleinen Gefässe befallen waren: die Wände waren verdickt und liessen keine Differenzirung mehr erblicken. Besonders im Bereich der Gummata bildeten die kleinen Gefässe eine charakteristische Structur: fast homogene Wände mit dünnen schwach gefärbten Faserchen; Zellen waren in diesen Wänden schwer zu finden. Phlebitis obliterans habe ich nicht gefunden. Ich habe auch keine Heubner'schen Arteriome gesehen, wie sie Orlowski und ich (l. c.) beschrieben haben. Von den grösseren Gefässen war vorwiegend die Arteria spinalis anterior specifisch erkrankt. Die Intima war deutlich verdickt, in den Spalten der Externa sah man hier und da kleinzellige Agglomerate (siehe oben); bis zum Verschluss des Lumens ist es aber nirgends gekommen, was sich übrigens durch die Localisation des Processes vorwiegend in den Häuten und Wurzeln der Hinterseite des Rückenmarks erklären lässt.

1) Kopczyński St., Przyczynek do symptomatologii i anatomii patologicznej przymiotu mózgu, oraz slow kilka o t. z. pseudo-paralysis luetica. - Sonderabdruck aus der "Medycyna“. Warschau 1899. - Zur Kenntniss der Symptomatologie und pathologischen Anatomie der Lues cerebri. - Deutsche Zeitschr. f. Nervenheilk. 1901. Bd. XX. 
In der Vena spinalis anterior habe ich hier und da wieder erweiterte Intimaspalten gefunden. Eine intensive Erkrankung der Rückenmarksarterien in einem Fall von Pseudotabes syphilitica hat Oppenheim bei Tabes und Rückenmarkssyphilis, Pick (siehe unten) und Goldflam (Fall 7) beschrieben.

Die Wurzeln der Cauda equina waren fast alle degenerirt. Dagegen war an der Peripherie rechts der N. tibialis ganz normal und nur links war der N. tibialis u. N. peroneus ausgesprochen degenerirt (s. Fig. 1-3). Goldflam (1. c.) hat in seinem Fall 10 trotz grosser Veränderungen der Wurzeln die peripheren Nerven fast ganz normal gefunden. Die Degeneration der Nerven links hing zweifelsohne damit zusammen, dass diese Nerven hier direct in die oben beschriebenen tiefen Brandwunden versenkt waren. Die Degeneration hatte einen vorwiegend parenchymatösen, nicht entzündlichen Charakter. In einigen Ulcerationen sah man die Nerven und Gefässe am Boden als dunkle Fäden verlaufen. Die Thatsache, dass der rechte $\mathrm{N}$. tibialis ganz normal war, spricht ganz entschieden gegen die Annahme einer multiplen Neuritis. An eine solche Nervenerkrankung könnte man aber denken angesichts der umgrenzten multiplen brandigen Stellen. Eine Polyneuritis kann den acuten Decubitus, die symmetrische Gangrän (Morbus Raynaud), Mal perforant du pied, Pemphigus und andere trophische Störungen verursachen. ${ }^{1}$ )

In unserem Falle aber, angesichts des normalen Zustandes des rechten $\mathrm{N}$. tibialis und des starken Befallenseins der Cauda equina, müssen wir die polyneuritische Aetiologie dieser Gangrän fallen lassen sowie auch die zwar selten vorkommende Rückenmarksaffection in Folge einer Polyneuritis, die sogenannte Myeloneuritis.

Schon beim Schneiden des aus der Müller'schen Flüssigkeit herausgenommenen Rückenmarks ist uns die starke Degeneration der Hinterstränge anfgefallen, so dass das Präparat anfänglich den Eindruck einer typischen Tabes machte.

Wenn wir uns an die fehlenden Patellar- und Achillessehnenreflexe erinnern, an die Schmerzen im ganzen Körper, oft von fliegender Beschaffenheit, an die In continentia urinae, die träge Pupillenreaction auf Lichteinfall gegen das Ende der Krankheit, so wird es uns gar nicht wundern, dass wir bei der makroskopischen Betrachtung und sogar am Anfang der mikroskopischen Untersuchung an Tabes gedacht haben. Die Untersuchung der unteren Rückenmarksabschnitte hat uns zwar gezeigt, dass wir es mit Syphilis des Lumbosacralabschnittes

1) Remak und Flatau, Neuritis und Polyneuritis (bei Nothnagel). Wien 1899. S. $40 \mathrm{ff}$. 
Syphilis des Rückenmarks u. seiner Häute in d. Lumbosacralgegend. 199

und der Cauda equina zu thun haben, sie konnte aber nicht die Möglichkeit der Coexistenz dieser beiden Affectionen, Lues und Tabes, ausschliessen. Diese etwas verwickelte Frage bedarf einer eingehenden Erörterung.

Die Pathogenese der Tabes hat jetzt eine umfangreiche Literatur. Sehmaus hat in seiner werthvollen Arbeit ${ }^{1}$ ) eine kritische Betrachtung einzelner, mehr oder weniger glücklich erdachter Hypothesen und Theorien geliefert. Er fasst sie folgendermassen zusammen: Tabes ist eine Erkrankung der Hinterwurzeln, die sich allmählich segmentweise entwickelt; sie befällt anfangs nur eine bestimmte Art von Fasern der Hinterwurzeln (elective Wurzelerkrankung), entsprechend der Reihenfolge der Bekleidung der Axencylinder mit den Myelinscheiden im Verlauf des Fötallebens (Flechsig), um schliesslich die ganzen Hinterwurzeln einzunehmen; in den späteren Perioden der Tabes werden auch die sogenannten endogenen Fasern degenerirt und sogar die ventralen Hinterstrangfelder.

Von den verschiedenen Theorien, die das Entstehen dieser Krankheit erklären, wie die primäre Afficirung der Hirnhaut (Redlich-Obersteiner), der Wurzeln (Nageotte), der Intervertebralganglien (Marie, Oppenheim), der peripheren Nerven (Takacs), scheint Schmaus für die Annahme einer primären Degeneration in den intraspinalen Theilen der Hinterwurzeln in Folge einer unbekannten Intoxication zu sein.

Die Beziehung der aufsteigenden Degeneration zu der tabischen besprechend, sagt Schmaus: „Es müsste schliesslich jede ausgeprägte Meningitis, wenn sie nur die dorsale Rückenmarksperipherie und die Gegend der hinteren Wurzeln mit betrifft, eine Tabes oder tabesähnliche Veränderungen zur Folge haben" (l. c. S. 180). An einer anderen Stelle sagt er, dass bei der Untersuchnng eines Tabesfalles, wo nur der Lumbal- und Sacralabschnitt einer intensiven Degeneration anheim gefallen sind, das anatomische Bild sich im Cervicalabschnitt garnicht von demjenigen unterscheiden wird, welches wir bei secundärer Degeneration zu sehen pflegen (S. 130).

Das Degenerationsgebiet hat in den Goll'schen Strängen eine flaschenförmige Gestalt; mit der Entfernung von der Peripherie wird es immer enger, um sich vor der Commissura posterior wieder zu erweitern.

Vertreter des Rückenmarkhautursprunges der Tabes sind Ober-

1) Schmaus, Vorlesungen über pathologische Anatomie des Rückenmarks. Wiesbaden 1901. 
steiner und Redlich ${ }^{1}$ ) gewesen, deren Meinung nach die bei Tabes entzündete Pia mater auf die Hinterwurzeln drückt, auf ibre Eingangsstelle in das Rückeumark (an der Einschnürungsstelle), wodurch eine aufsteigende Degeneration verursacht wird. Die genaueren Untersuchungen haben ergeben, dass diese Theorie zu sehr hypothetisch ist, da man in vielen Fällen von Tabes gar keine Veränderungen an der Rückenmarkshaut findet, da man weiter bei beiderseitig ungleichmässiger Erkrankung der Pia mater öfter einseitige, asymmetrische aufsteigende Degeneration finden müsste, die Tabes aber, abgesehen von nur. wenigen Ausnahmen, sich immer typisch symmetrisch entwickelt und schliesslich da die Tabes eine diffuse Erkrankung darstellt und oft die Kopfnerven, z. B. den Opticus mitbefällt, worauf die Hirnhaut sicher keinen Einfluss haben kann.

Trotz dieser Hinfälligkeit der Markhauthypothese hat man in der letzten Zeit begonnen, mehr auf den Zustand der Rückenmarkshäute bei Tabes zu achten, und es ist in einer ganzen Reihe von Fällen zu Genüge nachgewiesen worden, dass ein gleichzeitiges Vorkommen von beiden Affectionen ganz gut möglich ist: der Tabes und einer Rückenmarksentzündung syphilitischer Natur. $\mathrm{Ob}$ man hier mit zweierlei Gift zu thun hat, von welchem das eine den Schwund gewisser Fasersysteme im Rückenmark verursacht, das andere aber einen Reiz auf die Meningen und die Intima der Gefässe ausübt, lässt sich heute nicht mit Sicherheit entscheiden.

Andererseits sind in der letzten Zeit Fälle von syphilitischer Spinalaffection beschrieben worden, welche intra vitam unter dem Bilde der Tabes verliefen, mit dem Unterschiede, dass die Intensität der Symptome grosse Schwankungen zeigte, so dass es mitunter auch zu einer erheblichen Besserung kam, besonders unter dem Einfluss der specifischen Therapie. Solche Fälle von Pseudotabes syphilitica, wie sie heute genannt werden, sind klinisch sehr oft beschrieben worden: Orlowski (1. c.) citirt 3 solche Fälle (54, 55. und 56. Beobachtung), Gajkiewicz auch $3(48,49,50)$; Sectionsfälle sind von Oppenheim, Eisenlohr, Ewald, Siemerling und Valentin beschrieben worden.

Oppenheim ${ }^{2}$ ) beschreibt folgenden Fall: Es warde bei einer 31jährigen syphilitischen Frau im Jahre 1885 gefunden: lanzi-

1) Obersteiner und Redlich, Ueber Wesen und Pathogenese der tabischen Hinterstrangsdegeneration. Arbeiten aus dem Institut für Anatomie und Physiologie des Centralnervensystems. Leipzig und Wien 1894.

2) Oppenheim, Ueber einen Fall von syphilitischer Erkrankung des centralen Nervensystems, welche vorübergehend das klinische Bild der Tabes dorsalis vortäuschte. Berliner klinische Wochenschrift 1888. Nr. 53. 
Syphilis des Rückenmarks u. seiner Häute in d. Lumbosacralgegend. 201

nirende Schmerzen, aufgehobene Patellarreflexe, Romberg, reflectorische Pupillenstarre, Lähmung der $\mathrm{Nn}$. o culomotorii und accessorii Willisii, Störungen der Hautsensibilität, erschwerte Miction und Erbrechen. Es wurde Tabes diagnosticirt. Besserung nach einer Reihe von Inunctionen. Im Jahre 1886 Verschlimmerung; fehlende Patellarreflexe und gleichzeitig Fussclonus. Im nächsten Jahre Patellarreflexe bedeutend verstärkt, spastische Lähmung der beiden unteren Extremitäten. Ein Jahr später ergab die Autopsie Folgendes: ein Erweichungsherd im Nucleus lentiformis, specifische Veränderungen in den Hirngefässen, Pachymeningitis interna chronica et Arachnitis gummosa beginnend von der Gegend der mittleren Dorsalsegmente bis hinab zur Hälfte der Lumbalintumescenz; im unteren Dorsalabschnitt Infiltrate, die von der Peripherie ausgehend in das Innere der Rückenmarkssubstanz hineindringen; aufsteigende und absteigende Degeneration; die Hinterwurzeln des Lumbalabschnittes von gummösem Gewebe umgeben und stark degenerirt.

Eisenlohri) führt 2 Fälle an mit Lues in der Anamnese, wo intra vitam Tabes diagnosticirt wurde, wegen des höchst typischen klinischen Bildes. Post mortem wurde nicht die erwartete Systemdegeneration, sondern Meningitis spinalis syphilitica mit Gummata und eine secundäre Affection der Hinterstränge gefunden.

Im Falle Ewald ${ }^{2}$ ) fand man bei einem 42jährigen Kranken, der seit einem Jahr an lanzinirenden Schmerzen der unteren Extremitäten litt, Romberg, Westphal, Argyll-Robertson, Pupillendifferenz, Störungen der Haut-, Temperatur- und Muskelsensibilität, Arthropathie im linken Knie. Bei der Autopsie wurde garnicht das klassische tabische Entartungsbild gefunden, sondern ein subarachnoidaler Bluterguss längs des Rückenmarks, eine gummöse Entzündung der Pia und Arachnoidea, Verdickung der Bindegewebssepta; localisirte kleinzellige lnfiltration vorwiegend der Hinterstränge, Arteriitis et Phlebitis obliterans.

Die Bemühungen von Kahler und Buttersack eine besondere Form der Spinalsyphilis zu unterscheiden - die sogenannte multiple syphilitische Wurzelneuritis - sind erfolglos geblieben. Man hat sich nämlich mehrfach überzeugt, dass die Pia und Arachnoidea fast

1) Eisenlohr, Zur Pathologie der syphilitischen Erkrankungen der Hinterstränge des Rückenmarks. - Festschrift zur Eröffnung des Krankenhauses Hamburg-Eppendorf. 1889. (Cit. bei Nonne.)

2) Ewald, Ein unter dem klinischen Bilde der Tabes verlaufender Fall von syphilitischer Rückenmarkserkrankung. Berl. klin. Woch. 1893. Nr. 12. 
immer gleichzeitig mitafficirt sind. Die Nervenwurzeln sind in solchen Fällen, wie das auch in dem unsrigen zu sehen ist, entweder in die gummöse Masse eingehüllt, oder sie werden von narbigem Gewebe comprimirt, was natürlich zu secundären Degenerationen führen muss.

Klinisch prädominiren in solchen Fällen die Schmerzen. Derartige Beobachtungen haben mit mikroskopischer Untersuchung Pick, Dinkler, Brasch, Cassirer, Schmaus, Marinesco, Nageotte, Nonne, Minor, Sachs, Hoffmann-Kuh beschrieben.

Pick $\left.{ }^{1}\right)$ hat einen Fall veröffentlicht, welcher intra vitam das Bild der typischen Tabes bot, wo er bei der Autopsie neben der klassischen Hinterstrangdegeneration noch eine Verdickung der Meningen fand an der Basis cerebri und am Rückenmark. Besonders starke Meningealaffection mit Verkäsungsmassen fand er in den mittleren Partien des Dorsalabschnittes; ausserdem waren ausgesprochene specifische Veränderungen in der Arteria spinalis anterior vorhanden.

Dinkler ${ }^{2}$ ) hat im Jahre 1893 folgenden Fall beschrieben: Ein 42 jähriger Ingenieur hat sich vor 16 Jahren einen voraussichtlich harten Schanker zugezogen; im 36. Lebensjahre hatte er lanzinirende und reissende Schmerzen in den Beinen und in der Brust, im 39. das Gefühl von Ameisenkriechen unter den Fusssohlen, erschwerte Miction, Abnahme der geschlechtlichen Potenz. Objectiv wurden festgestellt: Myosis, reflectorische Pupillenstarre, Hypalgesie mit deutlich verzögertem Leitungsvermögen in den unteren Extremitäten, Hyperästhesie des Brustkastens, ungleichmässige Patellarreflexe, Fehlen des einen und Abschwächung des anderen Achillessehnenreflexes. Nach einigen Monaten Tod in Folge einer Hirnblutung. Die Autopsie ergab das klassische Entartungsbild der Hinterstränge in ihrem ganzen Verlauf und ausserdem eine gummöse Infiltration der Meningen, besonders in der Dorsalgegend und ausgeprägte, specifische Veränderungen der basalen Hirnarterien (Endarteriitis obliterans). In seinen Schlüssen bemerkt der Verfasser beiläufig, dass Tabes dorsalis und Syphilis viel öfter zusammen zu fallen scheinen, als das sonst vermuthet wird. In seiner Arbeit citirt er noch 5 Fälle ans Beobachtungen anderer A utoren von Tabes mit deutlicher Syphilis, darunter die Fälle von Hoffmann-Kuh und Minor.

Im Jahre 1900 veröffentlichte Dinkler ${ }^{3}$ ) zwei Fälle von Tabes

1) Pick, Tabes und Meningitis syphilitica. Festschrjft zu Ehren von F. J. Pick. 1898. Wien und Leipzig. (Cit. bei Nonne.)

2) Dinkler, Tabes dorsalis incipiens mit Meningitis spinalis syphilitica. Deutsche Zeitschr. f. Nervenheilk. 1893. Bd. 13.

3) Derselbe, Zur Aetiologie und pathol. Anat. der Tabes dorsalis. Deutsche Zeitschr. f. Nervenheilk. 1900, Bd. 18. 
Syphilis des Rückenmarks u. seiner Häute in d. Lumbosacralgegend. 203

und einen von Meningitis syphilitica und kommt zum Schluss, dass man bei genauerer Untersuchung verhältnissmässig oft Veränderungen in den Meningen bei Tabes dorsalis finden kann.

Ob nun aber in solchen Fällen eine Combination von Tabes und Meningitis syphilitica vorliegt, oder ob es sich um eine andersartige gegenseitige Beziehung dieser Erkrankungen handelt, will der Verfasser nicht entscheiden.

Hoffmann-Kuh haben bei einem 36jährigen vor 16 Jahren mit Lues inficirten Manne im Jahre 1887 folgende Erscheinungen gefunden: ungleiche, träg reagirende Pupillen, Ataxie der unteren Extremitäten, fehlende Kniereflexe, Sensibjlitätsstörungen in den Beinen, später lanzinirende Schmerzen und tabische Athropathie im rechten Knie. Mors im Jahre 1889. Die Autopsie ergab neben den typischen tabischen Spinalveränderungen Meningitis cerebrospinalis, Veränderungen in den Gefässen und Hyperostosis cranii.

Minor hat bei der Autopsie einer 26jährigen Tabischen neben der typischen Hinterstrangdegeneration eine Endo- et Periarteriitis luetica der Hirngefässe mit nachfolgender Erweichung in der linken Hemisphäre gefunden.

Alle oben angeführten Fälle beweisen zur Genüge die Möglichkeit einer Combination von Tabes mit einer syphilitischen Erkrankung der Meningen, der Gefässe oder der Gehirnsubstanz.

Wenn wir nun unseren Fall kritisch betrachten wollen, so werden wir doch nicht mit Sicherheit den tabischen Process vollkommen in Abrede stellen dürfen, obgleich wir ihn für wenig wahrscheinlich halten. Sicher ist es aber, dass wir eine Meningomyelitis syphilitica vor uns gehabt haben, die vorwiegend in den unteren Rückenmarksabschnitten localisirt war.

Wir hatten es in unserem Fall zu thun vor allen Dingen mit einer intensiven Erkrankung sämmtlicher Meningen und Nervenwurzeln, besonders der hinteren in der Lumbosacralgegend und der Cauda equina.

Die Ausdehnung der specifischen Affection auf die Hinterwurzeln führte zum Bild der aufsteigenden Degeneration der Hinterstränge. Unser Fall erinnert in mancher Hinsicht an den von Eiseulohr ${ }^{1}$ ) im Jahre 1884 beschriebenen, welcher sich kurz gefasst folgendermassen darstellt:

1) Eisenlohr, Meningitis spinalis chronica der Cauda equina mit secundärer Rückenmarksdegeneration, wahrscheinlich syphilitischen Ursprungs. Neurologisches Centralbl. 1884. 
Der Kranke, ein 30jähriger Kaufmann, wurde vor 11. Jahren mit Lues inficirt. Seit $1 \mathrm{Jahr}$ unangenehme Empfindungen und Schmerzen in der Aftergegend; einige Monate später Schwäche der Beine, namentlich des linken, dann Obstruction und Incontinentia urinae.

Später Lähmung des rechten Beines, welche unter dem Einfluss der specifischen Behandlung etwas zurückging. Der Patellarreflex links schwach, rechts lebhaft, später beiderseits erloschen. Auf den hinteren und etwas auf den seitlichen Flächen der Oberschenkel und der Waden Anästhesie. Decubitusgeschwüre an den Hinterbacken und Trochanteren. Tod nach 1 1/2 Jahren. Bei der Obduction vor allen Dingen. Veränderungen in der Cauda equina: einzelne Nervenwurzeln auf dem Querschnitt wie zusammengeklebt durch Bindegewebszüge, welche von den verdickten und verklebten Meningen ausgehen. In den hinteren Partien der Cauda waren diese Schwarten stärker, die Septa breiter und dicker als vorne. Die Nervenfasern waren hinten fast alle, vorne zum grossen Theil degenerirt. Die Degeneration der Hinterwurzeln erstreckte sich bis zu den oberen Lubalsegmenten hinauf. Die Hinterstränge (blos die Goll'schen Funikeln) waren degenerirt auf der ganzen Länge des Rückenmarks bis zu den Nuclei funiculi gracilis hinauf. Die Degenerationsfigur, bis zur Commissura posterior reichend, keilförmig. Im N. ischiadicus waren auch dentlich ausgeprägte Veränderungen. Der Verfasser bemerkt, dass ihn diese Erscheinungen anfangs an das klinische Bild einer atypischen Tabes dorsalis denken liessen.

Die Degeneration der Hinterstränge betraf in unserem Fall vorwiegend die Goll'schen Stränge; in den Burdach'schen waren keine Degenerationsstreifen zu sehen, was auf eine segmentale Degeneration der mehr nach oben gelegenen Nerrenwurzeln hinweisen könnte. Die sogenannte Wurzeleintrittszone und die Seitenstränge waren in den oberen Rückenmarkssegmenten gut erhalten. Das ventrale Hinterstrangfeld pflegt bekanntlich erst in den Spätstadien der Tabes za degeneriren. In unserem Fall war dieses Feld ziemlich stark degenerirt, was öfter bei aufsteigenden Degenerationen beobachtet wird.

Die Clarke'schen Säulen sehen im anatomischen Bild dex Tabes bedeutend blässer aus, als auf normalen Präparaten, was nicht durch den Zellenschwund dieser Stränge, sondern dureh die Atrophie des zarten Netzes von Verzweigungen und Seitenästen verursacht wird, welche von den Hinterwurzeln zu den Clarke'schen Zellen gelangen. Die Zellen selbst bleiben gewöhnlich intact und deswegen fehlt bei Tabes die Entartung der Kleinhirnbahnen, welche aus ihren Axencylindern bestehen. Die in unserem Fall vorhandene Degeneration im Bereich der Kleinhirnbahnen und der Gowers'schen Stränge ist 
Syphilis des Rückenmarks u. seiner Häute in d. Lumbosacralgegend. 205

auf allerdings geringfügige Veränderungen der Rückenmarkssubstanz selbst zurückzuführen. Ebenfalls spricht bei uns gegen die Annahme von Tabes die mehrere Wochen vor dem Tode gefundene leichte Neuritis optica. In dem oben angeführtem Fall von Pick hat sich aber dem klinischen Bilde der Tabes auch eine Neuritis optica angeschlossen als Ausdruck einer gleichzeitigen syphilitischen Erkrankung des Nervensystems.

Das wichtigste und früheste organische Symptom war bei unserer Patientin das Fehlen der Patellar- und Achillessehnenreflexe. Es ist schwer zu entscheiden, ob es sich hier um eine geringfügige Störung der Blutversorgung des Rückenmarks handelte mit functionellen Veränderungen und Unterbrechen des Leitungsvermögens im Reflexbogen, oder ob schon damals die Infiltration der Meningen des Lumbalabschnittes auf die Hinterwurzeln der entsprechenden Höhe übergegriffen hatte. Jedenfalls mahnte schon damals ein so empfindliches Reagens, wie das Westphal'sche Symptom, an die Nothwendigkeit einer energischen specifischen Kur, welche der Kranken noch das Leben retten könnte.

Unsere Patientin unterlag der Rückenmarkssyphilis in ihrem 20. Lebensjahre; wann sie inficirt worden ist, ist unbekannt. Auf das Alter von 20-40 Jahren entfällt nach den meisten Verfassern der grösste Procentsatz syphilitischer Erkrankungen. Was das Syphilisstadium anbetrifft, in welchem die Spinalerscheinungen aufzutreten pflegen, so hat hier natürlich die Eintheilung in primäre, secundäre und tertiäre Lues gar keine Bedeutung. Es sind zahlreiche Beobachtungen bekannt, wo Erscheinungen von Spinalerkrankungen schon wenige Monate nach der Infection aufgetreten sind. Von 18 Beobachtungen von Goldflam sind in 13 Fällen die ersten Symptome vor Ablauf des ersten Jahres aufgetreten; unter den 60 Fällen von Orlowski entfallen 23 (40 Proc.) auf die ersten zwei Jahre, im Fall VI ist das Spinalleiden 3 Monate nach der Infection zusammen mit dem allgemeinen Hautexanthem aufgetreten. Es kommt also nicht an auf die periodische Reiheufolge der Erscheinungen, sondern auf die Intensität der Giftwirkung und die mehr oder weniger lebhafte Reaction des Organismus. Glücklicherweise ist das syphilitische Virus bei Weitem nicht so gefährlich, denn nach einer, zwar nicht besonders genauen, Statistik (Hielmann) erkrankt auf 10000 Syphilitiker blos einer an einem specifischen Leiden des Nervensystems.

Zum Schluss halte ich es für eine angenehme Pflicht, dem Collegen Dr. E. Flatau auch an dieser Stelle meinen innigsten Dank für seine werthvolle Leitung und Unterstützung anszusprechen. 


\section{Erklärnng der Figuren und Tafel II.}

Fig. 1. Nervus tibialis sinister (Querschnitt).

Fig. 2. N. tibialis sinister (Längsschnitt). Beide stark degenerirt.

Fig. 3. N. tibialis dexter (Querschnitt), fast normal.

Fig. 4. Höhe des dritten Sacralsegments mit Cauda equina, stark degenerírt.

Fig. 5. Höhe des sechsten Dorsalsegments, starke Degeneration der Hinterstränge, leichte Degeneration der Kleinhirnstränge und des Gowersbündels:

Fig. 6. Theil des Rückenmarks mit den Hüllen. m.s. Peripherie des Rückenmarks; $g l$. Neurogliaschicht auf der Peripherie des Rückenmarks; $w$ degenerirte Rückenmarkswurzel; $g$. gummöse Infiltration; $d$. verdickte Dura mater.

Fig. 1, 2, 3. Mikrophotographien, Leh $r$ ).

Fig. 4, 5, 6. Photographien der Zeichnungen nach den Präparaten (Dr.

Fig. 4 Vergrösserung $1: 6$,

Fig. $5 \quad " \quad 1: 8$,

Fig. $6 \quad 1: 16$.

Taf. II: Photographie der Leiche der Patientin mit den Geschwüren. 


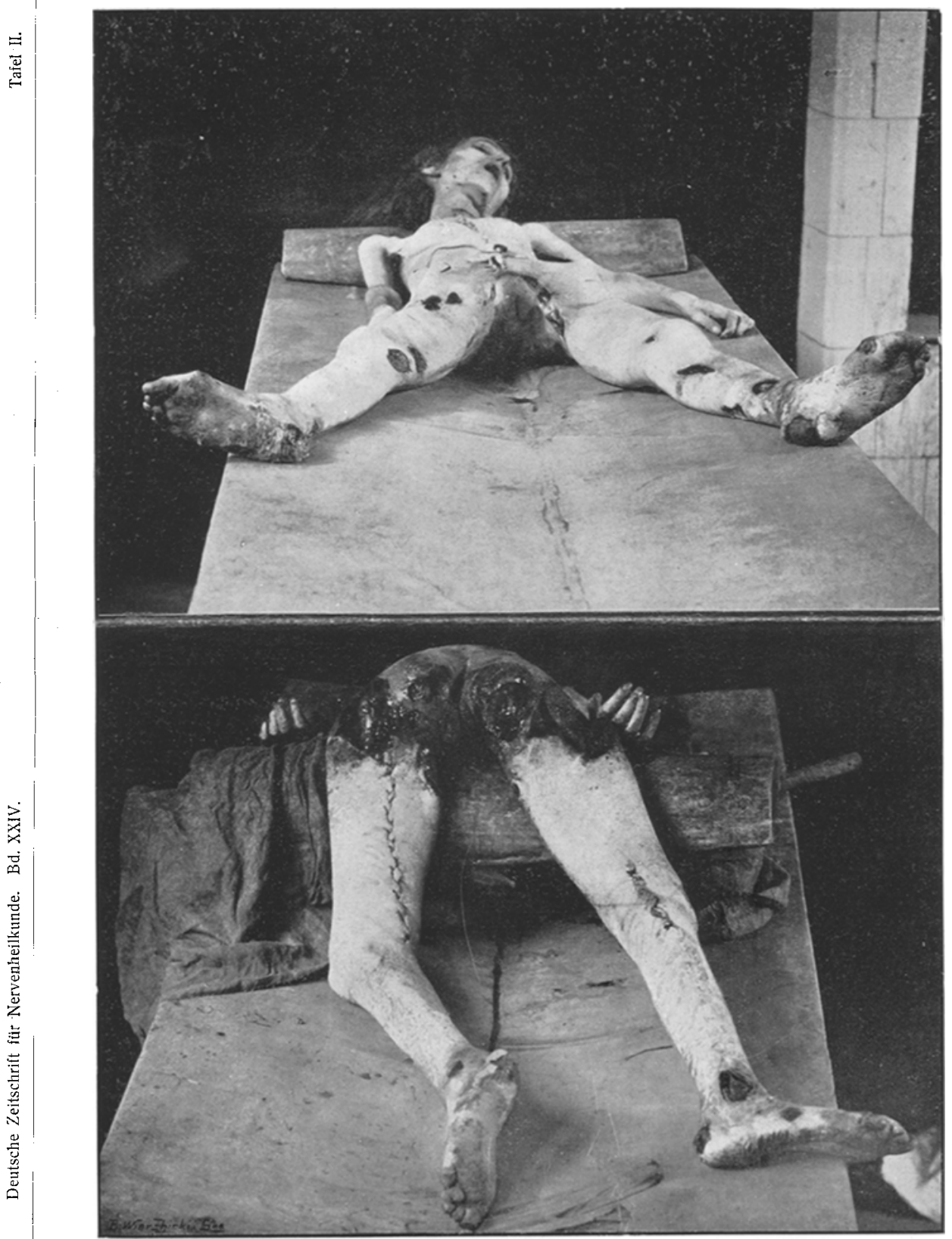

